\title{
An Eye-Tracking Assessment of Coronary Care Nurses during the Interpretation of Patient Monitoring Scenarios
}

\author{
Jonathan Currie $^{1}$, Raymond R Bond ${ }^{1}$, Paul McCullagh ${ }^{1}$, Pauline Black ${ }^{2}$, \\ Dewar D Finlay ${ }^{3}$, Aaron Peace ${ }^{4}$ \\ ${ }^{1}$ Computer Science Research Institute, Ulster University, N. Ireland \\ ${ }^{2}$ Institute of Nursing and Health Research, Ulster University, N. Ireland \\ ${ }^{3}$ Engineering Research Institute, Ulster University, N. Ireland \\ ${ }^{4}$ Clinical Translational Research and Innovation Centre, Altnagelvin Hospital, N. Ireland
}

\begin{abstract}
Introduction: It has yet to be determined whether visual attention, measured via eye tracking metrics (ETMs) can be indicative of performance level in coronary care nursing when interpreting patient vitals.

Methods: This study captures the visual attention of nurses when interpreting five scenarios using simulated text and vital signs. Baseline performance was marked using detailed criteria and scored 0-10. Self-rated confidence from 1-10 was also collected for each scenario. Cognitive workload was assessed by measuring a participant's heart rate and post-performance NASA$T L X$ responses. Eleven coronary care nurses were recruited providing 55 interpretations/observations in total. 45 of which, post data quality filtering, were used to analyse ETMs.

Results: Mean performance score $=6.86 \pm 1.50$ and mean confidence rating $=7.51 \pm 1.2$. A subset of ETMs significantly correlate with performance across all scenarios. Individual scenarios also provide significant correlations. Three of six regression models were statistically significant with $R^{2} \geq 0.5$.

Conclusion: Correlations between specific ETMs and performance have been found across all scenarios and for individual scenarios. Further work is needed to confirm the benefit of ETM in assessing simulation-based training performance.
\end{abstract}

\section{Introduction}

Patient safety and medical errors are a critical area of concern within healthcare [1]. Lack of knowledge and skill with clinical procedures, as well as decision-making can be significant factors with many of the errors that are reported [2].

Many healthcare tasks can be simulated using computer/web technology to provide trainees with a method to improve knowledge and skills [3]. Computerbased simulation still requires further research to demonstrate how it can provide a fully comparable training experience compared to other modalities, such as mannequin-based simulation, standardized patients etc.

Monitoring is a core competency in nursing involving surveillance of the patient and their physiological signals [4]. This is imperative for the early detection of patient deterioration [5]. There is a need for more consistent practice in patient surveillance [6] and effective assessment of patient vital signs [7].

Research into the understanding of visual attention during healthcare tasks has increased [8]-[10]. The mindeye hypothesis states that measurements of visual attention may indicate underlying cognitive activity [11].

The objective of this study was to assess the role of eye tracking metrics (ETMs) and other psychophysiological measurements as independent assessors for a trainee performing interpretation of patient vital signs [12]. This work contributes to the viability of automated simulation based assessment in nursing.

\section{Methods}

Eleven coronary care nurses were recruited during a one-day ECG seminar at Altnagelvin Hospital. They were asked to verbally interpret five different monitoring scenarios while being eye-tracked and wearing a heart rate (photo-plethysmography) monitor. Visual attention during the task was captured using the Tobii X60 eye tracker (tobiipro.com) at a recording frequency of $30 \mathrm{~Hz}$ and heart rate by wearing an Empatica E4 wristband (empatica.com). After performing the five interpretations, we asked each participant to complete a NASA-TLX (humansystems.arc.nasa.gov) survey, consisting of seven scaled questions related to task performance (e.g. mental demand, frustration etc.) to allow us to analyse their cognitive workload during the task. 


\subsection{Patient scenarios}

The vital signs scenarios were designed/validated by nursing experts (following work aimed at capturing these monitoring scenarios into digital format [13]) and then simulated using Laerdal's SimMan simulation software (laerdal.com). One minute of each set screen were recorded to use in the task. The patient vignettes settings were as follows: (1) medical ward, (2) cardiology ward, (3) surgical ward, (4) respiratory ward, (5) emergency department. Each scenario contained ten available marks for the interpretation.

Once the participant was ready, the next screen contained the patient's vital signs, for them to verbally interpret: Electrocardiogram and Heart Rate, Arterial Blood Pressure, Central Venous Pressure, Oxygen saturation, Respiratory Rate and Temperature. All provide areas of interest (AOI) for eye tracking metrics (ETM) used in the analysis, see Figure 1.

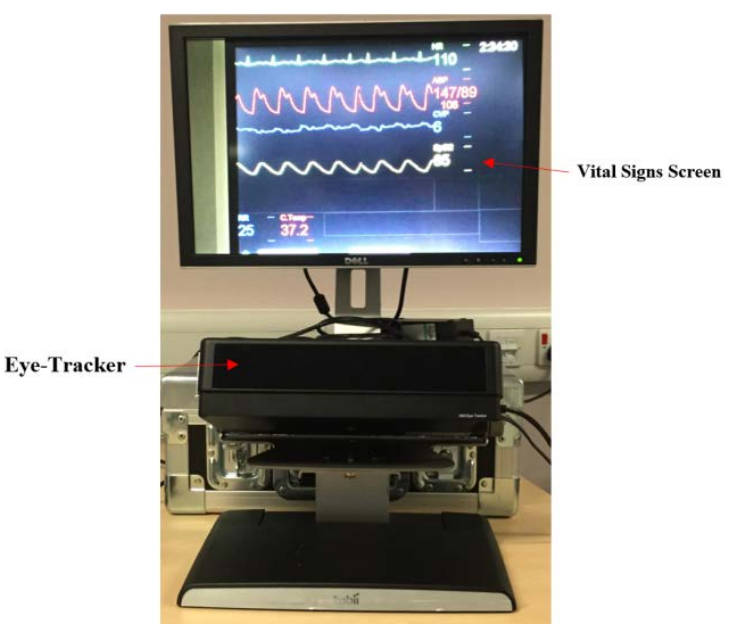

Figure 1. Tobii X60 Eye Tracker and Simulated Vital Signs Screen.

\subsection{Data analysis}

The AOIs that ETMs would perform measurement on were coded as such: Electrocardiogram = ECG, Heart Rate $=H R$, Arterial Blood Pressure* $=A B P+[W]$ or $[N]$, Central Venous Pressure* $=C V P+[W]$ or $[N]$, Oxygen Saturation $*=\mathrm{SpO}_{2}+[\mathrm{W}]$ or $[N]$, Temperature $=T E M P$, Respiratory Rate $=R E S P . *$ These vital signs have both a wave and numeric component, with $[\mathrm{W}]=$ Wave and $[N]$ $=$ Number, analysed separately.

We also wished to analyse visual attention spent on non AOI areas of the screen - as we saw this as potentially indicative of uncertainty. This is coded as $N O N-A O I$ and ETMs measure it the same way as the AOIs.
The ETMs provided by the Tobii eye tracking software (tobiipro.com) are shown in Table 1.

Table 1. Eye tracking metrics (ETMs) used in analysis

ETM Analysis $\begin{gathered}\text { Anseviation } \\ \text { Abbrevion }\end{gathered}$

\begin{tabular}{|c|c|c|}
\hline $\begin{array}{l}\text { Time to first } \\
\text { fixation (s) }\end{array}$ & TTFF & $\begin{array}{l}\text { Time elapsed before first } \\
\text { fixation on area of } \\
\text { interest. }\end{array}$ \\
\hline $\begin{array}{l}\text { First fixation } \\
\text { duration (s) }\end{array}$ & $F F D$ & $\begin{array}{c}\text { Time elapsed during first } \\
\text { fixation on area of } \\
\text { interest. }\end{array}$ \\
\hline $\begin{array}{l}\text { Fixations before } \\
\text { (number) }\end{array}$ & $F B$ & $\begin{array}{c}\text { Number of fixations } \\
\text { (elsewhere) before first } \\
\text { on area of interest during } \\
\text { viewing. }\end{array}$ \\
\hline $\begin{array}{l}\text { Fixation duration } \\
\text { (s) }\end{array}$ & $F D$ & $\begin{array}{l}\text { Mean elapsed time from } \\
\text { all fixations. }\end{array}$ \\
\hline $\begin{array}{l}\text { Fixation count } \\
\text { (number) }\end{array}$ & FC & $\begin{array}{c}\text { Total number of fixations } \\
\text { on area of interest. }\end{array}$ \\
\hline $\begin{array}{l}\text { Total fixation } \\
\text { duration (s) }\end{array}$ & TFD & $\begin{array}{l}\text { Total elapsed fixation } \\
\text { time on area of interest. }\end{array}$ \\
\hline Visit duration (s) & $V D$ & $\begin{array}{l}\text { Mean elapsed time from } \\
\text { first fixation inside area } \\
\text { of interest until eye gaze } \\
\text { exits. }\end{array}$ \\
\hline $\begin{array}{l}\text { Visit count } \\
\text { (number) }\end{array}$ & $V C$ & $\begin{array}{l}\text { Total number of visits on } \\
\text { area of interest. }\end{array}$ \\
\hline $\begin{array}{l}\text { Total visit } \\
\text { duration (s) }\end{array}$ & $T V D$ & $\begin{array}{l}\text { Total elapsed visit time } \\
\text { on area of interest. }\end{array}$ \\
\hline
\end{tabular}

Manual operator quality control procedures were applied for eye tracking recordings and those that fell below 50\% recording quality (e.g. participant looking away from the screen too much) were excluded from analysis. The analysis was performed using $\mathrm{R}$ programming in RStudio (rstudio.com) with core $\mathrm{R}$ functionality used for regression model building and with dplyr/tidyr, Hmisc and DAAG libraries used for data tidying, correlation testing and cross validating regression models respectively.

Our primary analysis here is to present moderate to strong correlating ETMs to performance score, both across all scenarios but also individual scenarios interpreted by participants. With significant correlations found we attempted simple linear or multiple linear regression to predict the score. Cross validation was performed on some of these models with mean squared error (MSE) given: due to limited sample size, only results presented are with regression models that produced $\mathrm{R}^{2}$ or Multiple $\mathrm{R}^{2} \geq 0.80$.

We also present correlations amongst NASA-TLX and heart rate monitoring for task cognitive loading.

\section{Results}

The nurses recruited had an age range 25-43 years, with a mean age of 32 years; experience range 5-17 years 
with a mean of 9 years. Table 2 provides performance and confidence scores across all scenarios by all participants.

Table 2. Participant Performance: total score and confidence.

\begin{tabular}{cccc} 
Measure & Mean \pm SD & Min & Max \\
\hline Total Score $(0-10)$ & $6.86 \pm 1.50$ & 4 & 10 \\
\hline Confidence (1-10) & $7.51 \pm 1.20$ & 5 & 10
\end{tabular}

\subsection{ETMs and performance score}

Due to data quality filtering (see 2.2.), two of eleven participants were removed, resulting in the removal of ten interpretations. This leaves 45 total observations to analyse. Significant correlations for ETMs and performance score across all scenarios are presented in Table 3. The most significant model $(\mathbf{p}=\mathbf{0 . 0 1})$ used three of the five available predictors, with Multiple $\mathbf{R}^{2}=\mathbf{0 . 3 4}$. Three-fold cross validation produced $\mathbf{M S E}=\mathbf{1 . 3 5}$. Figure 2 shows the cross validation prediction values against dataset performance scores.

\begin{tabular}{cccc}
\multicolumn{4}{c}{ Table 3. Correlating ETMs with score across all scenarios. } \\
AOI & ETM & $\boldsymbol{r}$ & p \\
\hline TEMP & TTFF & 0.44 & $<0.01$ \\
RESP & & 0.43 & $<0.01$ \\
\hline TEMP & $F B$ & 0.39 & $<0.05$ \\
\hline NON-AOI & $F F D$ & -0.35 & $<0.05$ \\
\hline TEMP & $V D$ & -0.31 & $<0.05$
\end{tabular}

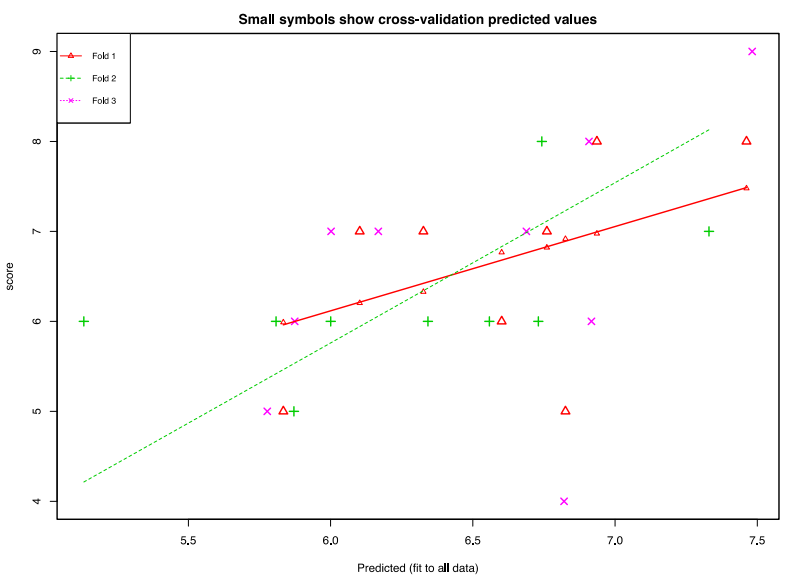

Figure 2. Predicting performance score across all scenarios using ETMs: three-fold cross validation plotted values (predictions) vs dataset scores

Correlation analysis of ETMs for the individual scenarios are shown in Table 4, including details of predictive model attempts.

Table 4. Correlating ETMs and score for each scenario, with simple linear or multiple linear regression models and cross-validation on models with $\mathrm{R}^{2} \geq 0.8$.

\begin{tabular}{|c|c|c|c|c|c|c|}
\hline & Scenario & AOI & ETM & $r$ & $\mathbf{p}$ & Predictive Model Details \\
\hline \multirow{2}{*}{1} & \multirow{2}{*}{ Medical Ward } & \multirow{2}{*}{ NON-AOI } & $F D$ & -0.71 & $<0.05$ & \multirow{2}{*}{$\begin{array}{l}\text { Most significant model }(\mathbf{p}=\mathbf{0 . 0 2}) \text { using } \\
\text { all available predictors with } \\
\text { Multiple } \mathbf{R}^{2}=\mathbf{0 . 7 2} \text {. }\end{array}$} \\
\hline & & & $F B$ & 0.68 & $<0.05$ & \\
\hline 2 & Cardiology Ward & \multicolumn{5}{|c|}{ No significant ETM correlations to performance score and no significant predictive regression models. } \\
\hline \multirow{3}{*}{3} & \multirow{3}{*}{ Surgical Ward } & RESP & & 0.80 & $<0.05$ & \multirow{3}{*}{$\begin{array}{l}\text { Most significant model }(\mathbf{p}=\mathbf{0 . 0 2}) \text { using } \\
1 \text { of the } 2 \text { available predictors with } \\
\mathbf{R}^{2}=\mathbf{0 . 6 4} \text {. }\end{array}$} \\
\hline & & $\mathrm{SpO}_{2}[\mathrm{~W}]$ & $T T F F$ & 0.70 & $<0.05$ & \\
\hline & & TEMP & $F F D$ & 0.68 & $<0.05$ & \\
\hline \multirow{8}{*}{4} & \multirow{8}{*}{$\begin{array}{l}\text { Respiratory } \\
\text { Ward }\end{array}$} & & $F B$ & -0.82 & $<0.01$ & \multirow{4}{*}{$\begin{array}{l}\text { Most significant model }(\mathbf{p}=\mathbf{0 . 0 2}) \text { using } \\
2 \text { from } 8 \text { available predictors with a } \\
\text { Multiple } \mathbf{R}^{\mathbf{2}}=\mathbf{0 . 8 3} \text {. }\end{array}$} \\
\hline & & $A B P[W]$ & TTFF & -0.78 & $<0.01$ & \\
\hline & & $N O N-A O I$ & $F F D$ & -0.80 & $<0.01$ & \\
\hline & & \multirow{4}{*}{$C V P[N]$} & $F D$ & -0.78 & $<0.01$ & \\
\hline & & & TFD & -0.77 & $<0.05$ & \multirow{4}{*}{$\begin{array}{l}\text { 3-fold cross validation produced } \\
\mathbf{M S E}=\mathbf{0 . 3 2}\end{array}$} \\
\hline & & & TVD & -0.77 & $<0.05$ & \\
\hline & & & $F C$ & -0.68 & $<0.05$ & \\
\hline & & TEMP & $V C$ & 0.69 & $<0.05$ & \\
\hline \multirow{7}{*}{5} & \multirow{7}{*}{$\begin{array}{l}\text { Emergency } \\
\text { Department }\end{array}$} & \multirow{5}{*}{$C V P[W]$} & TFD & -0.91 & $<0.001$ & \multirow{4}{*}{$\begin{array}{l}\text { Most significant model }(\mathbf{p}<\mathbf{0 . 0 0 1}) \\
\text { using } 1 \text { from } 7 \text { available predictors } \\
\text { with } \mathbf{R}^{2}=\mathbf{0 . 8 3}\end{array}$} \\
\hline & & & $T V D$ & -0.90 & $<0.01$ & \\
\hline & & & $V D$ & -0.77 & $<0.05$ & \\
\hline & & & $F C$ & -0.68 & $<0.05$ & \\
\hline & & & $V C$ & -0.71 & $<0.05$ & \multirow{3}{*}{$\begin{array}{l}\text { 3-fold cross validation produced } \\
\text { MSE }=\mathbf{0 . 2 5}\end{array}$} \\
\hline & & \multirow{2}{*}{$A B P[W]$} & TFD & -0.79 & $<0.01$ & \\
\hline & & & $T V D$ & -0.77 & $<0.05$ & \\
\hline
\end{tabular}




\subsection{NASA-TLX and heart rate}

Fourteen significant correlating variables were found amongst NASA-TLX (both total score overall and individual questions responses), heart rate (bps), their mean confidence rating (1-10) and other demographic information. Neither NASA-TLX or heart rate values correlate strongly with performance score, see Table 5.

Table 5. Correlations between NASA-TLX and non-ETM

\begin{tabular}{|c|c|c|c|}
\hline Var. i & Var. ii & $r$ & p \\
\hline \multirow{6}{*}{$\begin{array}{l}\text { TLX: Mental } \\
\text { Demand }\end{array}$} & TLX: Effort & 0.61 & $<0.001$ \\
\hline & TLX: Total & 0.67 & $<0.001$ \\
\hline & Age (yrs.) & -0.51 & $<0.001$ \\
\hline & Experience (yrs.) & -0.56 & $<0.001$ \\
\hline & Initial Heart Rate & -0.73 & $<0.001$ \\
\hline & Mean Heart Rate & -0.63 & $<0.001$ \\
\hline \multirow{3}{*}{$\begin{array}{l}\text { TLX: Temporal } \\
\text { Demand }\end{array}$} & TLX: Total & 0.73 & $<0.001$ \\
\hline & Age (yrs.) & -0.62 & $<0.001$ \\
\hline & Experience (yrs.) & -0.64 & $<0.001$ \\
\hline \multirow{2}{*}{ TLX: Effort } & Initial Heart Rate & -0.73 & $<0.001$ \\
\hline & $\Delta$ Heart Rate & 0.53 & $<0.001$ \\
\hline TLX: Frustration & Final Heart Rate & 0.55 & $<0.001$ \\
\hline TLX: Total & Experience (yrs.) & -0.56 & $<0.001$ \\
\hline Confidence & Mean Heart Rate & -0.55 & $<0.001$ \\
\hline
\end{tabular}

\section{Discussion and conclusions}

This study provides significant correlating ETMs to task performance. TTFF for both (1) TEMP and (2) RESP are the most significant with score across all scenarios. Notably both are situated at the bottom of the screen suggesting better performers digest information from AOIs above for longer than poorer performers. Notably CVP $[W] /[N]$ are strong negative correlators to score in scenarios four and five. In both scenarios CVP is normal with no significant relation to recommendations that would achieve higher scores. Eye-tracking appears to see a link between those who ignore this AOI and better performance. NASA-TLX and heart rate also provide some significant correlations with each other. Notably that both mental demand and effort have a negative but strong correlation with participant initial heart rate.

The results provide indicators for further research into ETMs and performance in simulated training for vital sign interpretation. A larger dataset could provide significantly more insight to the cognitive process of this task and lead to validated metrics for assessment of trainee nurses. Confirmation of discriminating metrics could lead to automatic classification between performance level - either low/high performance or at least uncertain/confident interpretations. The former providing a method to efficiently quantify training level of trainee, the latter to assess uncertainty of nurses at the bedside.

\section{Acknowledgements}

This research was funded by the Department of Employment and Learning (DEL), Northern Ireland.

\section{References}

[1] L. T. Kohn, J. M. Corrigan, and M. S. Donaldson, To Err is Human: Building a Safer Health System. National Academies Press (US), 2000.

[2] J. Zhang, V. L. Patel, and T. R. Johnson, "Medical error: is the solution medical or cognitive?," J. Am. Med. Inform. Assoc., vol. 9, no. 6 Suppl, pp. S75-7, 2002.

[3] F. E. Bogossian, S. J. Cooper, R. Cant, J. Porter, and H. Forbes, "A trial of e-simulation of sudden patient deterioration (FIRST2ACT $\mathrm{WEB}^{\mathrm{TM}}$ ) on student learning,” Nurse Educ. Today, vol. 35, no. 10, pp. e36-e42, 2015.

[4] M. Elliott and A. Coventry, "Critical care: the eight vital signs of patient monitoring.," Br. J. Nurs., vol. 21, no. 10, pp. 6215, 2012.

[5] A E. Rogers, G. E. Dean, W.-T. Hwang, and L. D. Scott, "Role of registered nurses in error prevention, discovery and correction,” Qual. Saf. Heal. Care, vol. 17, no. 2, pp. 117121, 2008.

[6] S. Osborne, C. Douglas, C. Reid, L. Jones, G. Gardner, “The primacy of vital signs - Acute care nurses' and midwives' use of physical assessment skills: A cross sectional study," Int Nur. Stud vol. 52, pp. 951-962, 2015.

[7] M. A. F. Pimentel, D. A. Clifton, L. Clifton, P. J. Watkinson, and L. Tarassenko, "Modelling physiological deterioration in post-operative patient vital-sign data," Med. Biol. Eng. Comput., vol. 51, no. 8, pp. 869-877, 2013.

[8] A. Fong, D. J. Hoffman, A. Zachary Hettinger, R. J. Fairbanks, and A. M. Bisantz, "Identifying visual search patterns in eye gaze data; gaining insights into physician visual workflow," J. Am. Med. Informatics Assoc., p. ocv196, Apr. 2016.

[9] S. Zhou, R. Gali, M. Paasche-Orlow, and T. W. Bickmore, "Afraid to ask: proactive assistance with healthcare documents using eye tracking," Proc. Ext. Abstr. 32nd Annu. ACM Conf. Hum. factors Comput. Syst. - CHI EA '14, pp. 1669-1674, 2014.

[10] P. O’Meara, G. Munro, B. Williams, S. Cooper, F. Bogossian, L. Ross, L. Sparkes, M. Browning, and M. McClounan, "Developing situation awareness amongst nursing and paramedicine students utilizing eye tracking technology and video debriefing techniques: A proof of concept paper.," Int. Emerg. Nurs., vol. 23, pp. 94-9, 2015.

[11] M. P. Stiegler and D. M. Gaba, "Eye Tracking to Acquire Insight Into the Cognitive Processes of Clinicians," Simul. Healthc. J. Soc. Simul. Healthc., vol. 10, pp. 329-330, 2015.

[12] J. Currie, R. Bond, P. McCullagh, P. Black, and D. Finlay, "Using Eye-Tracking Technology to Capture Visual Attention during Interpretation of a Simulated Bedside Monitor," in Irish Human Computer Interaction, Dublin. iHCI, 2015, p. 1.

[13] J. Currie, R. R. Bond, P. McCullagh, P. Black, and D. D. Finlay, "VitalSimML - A well-formed data structure to Capture Patient Monitoring Scenarios to facilitate the training of nurses via computer-based simulation," in Computing in Cardiology, 2016, vol. 42, pp. 421-424.

Address for correspondence:

Jonathan Currie, Ulster University, Shore Road, Newtownabbey, BT37 0QB, [currie-j@email.ulster.ac.uk] 